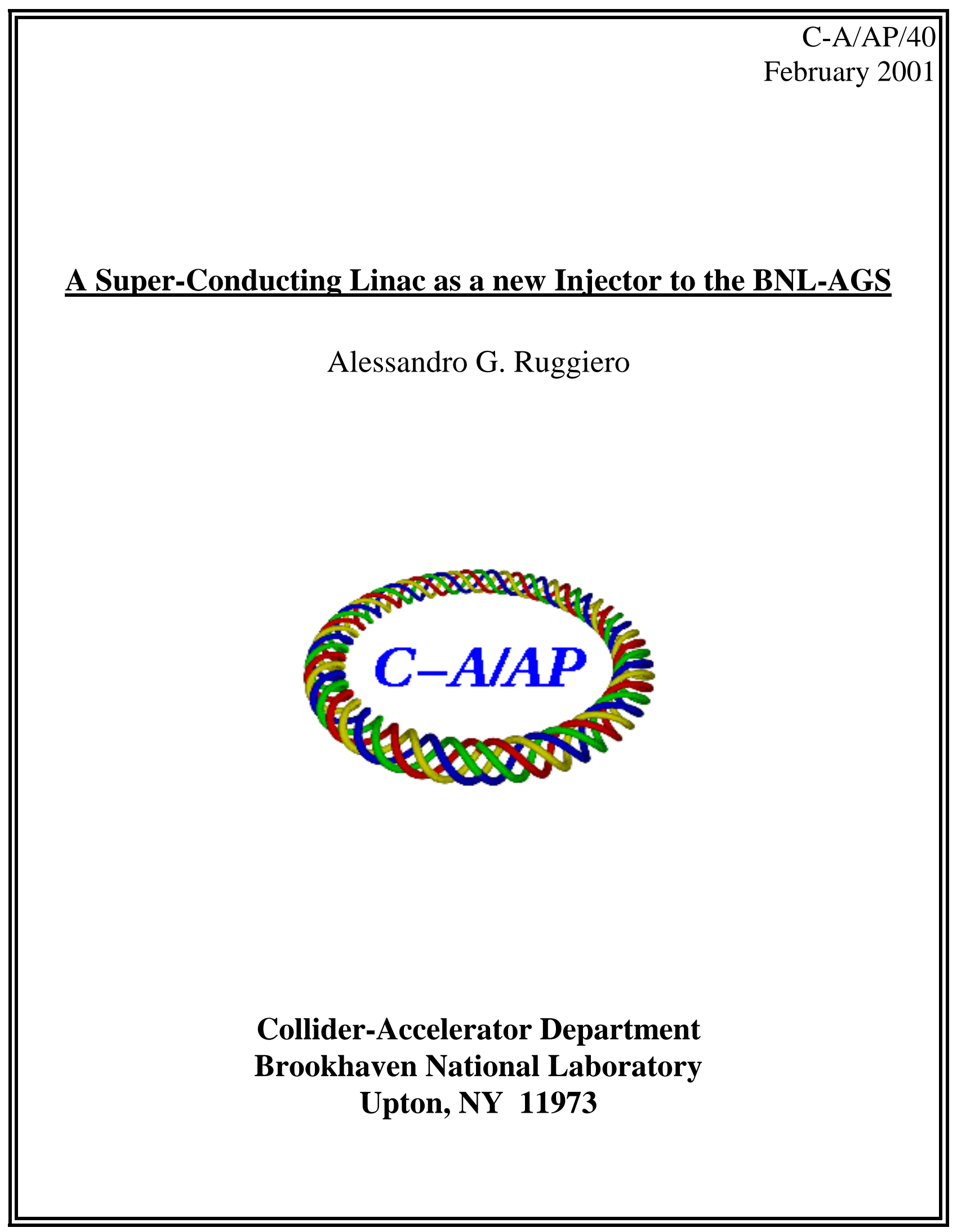




\title{
A Super-Conducting Linac as a new Injector to the BNL-AGS ${ }^{*}$
}

\author{
Alessandro G. Ruggiero \\ Brookhaven National Laboratory, PO BOX 5000; Upton, NY 11973 \\ February 16, 2001
}

\begin{abstract}
This paper reports on the feasibility study of a proton Super-Conducting Linac (SCL) as a new injector to the Alternating Gradient Synchrotron (AGS) of the Brookhaven National Laboratory (BNL). The Linac beam energy is $1.5 \mathrm{GeV}$. The beam intensity is adjusted to provide an average beam power of $1 \mathrm{MW}$ at the top energy of $24 \mathrm{GeV}$. The repetition rate of the Linac-AGS facility is one beam pulse per every 0.4 -second.
\end{abstract}

\section{Introduction}

It has been proposed to upgrade the Alternating Gradient Synchrotron (AGS) accelerator complex to provide an average proton beam power of $1 \mathrm{MW}$ at the energy of $24 \mathrm{GeV}$. The facility can be used as a proton driver for the production of intense muon and neutrino beams. The upgrade requires operation of the accelerator at the rate of 2.5 cycles per second, and a new injector for an increase of the AGS beam intensity to $1 \times 10^{14}$ protons per pulse, an increase of about $30 \%$ over the present mode of operation. Other upgrade schemes have also been investigated earlier [1,2]. The present injector made of the $200-\mathrm{MeV}$ Linac and of the $1.5-\mathrm{GeV}$ AGS-Booster will not be able to fulfill the goals of the upgrade.

The proposed new injector; described in this technical report, is a $1.5 \mathrm{GeV}$ SuperConducting Linac with an average output beam power of $62.5 \mathrm{~kW}$. The beam energy is determined by the capability to limit beam losses due to stripping of the negative ions that are used for multi-turn injection into the synchrotron, to reduce the effects of the space-charge forces, to accommodate adequately the beam transverse size in the aperture of the accelerator, and to fit the length of the accelerator on the real estate next to the AGS. The Linac is pulsed and the duty cycle is about a half percent.

The paper describes the preliminary design of the Super-Conducting Linac. It is composed of four parts, as shown in Figure 1: a Front End; that is a 37.5-mA negativeion source, followed by a 750-keV RFQ; a Low-Energy (LE) section that accelerates protons to $400 \mathrm{MeV}$, and the two sections of the Super-Conducting Linac proper. The two sections, each with its own energy range, and different cavity-cryostat arrangement; are labeled: Medium-Energy (ME), for acceleration to $800 \mathrm{MeV}$, and High-Energy (HE) that accelerates to $1.5 \mathrm{GeV}$. A short transport line that takes the beam for multi-turn injection into the AGS then follows the Linac.

\footnotetext{
* Work performed under the auspices of the US Department of Energy.
} 


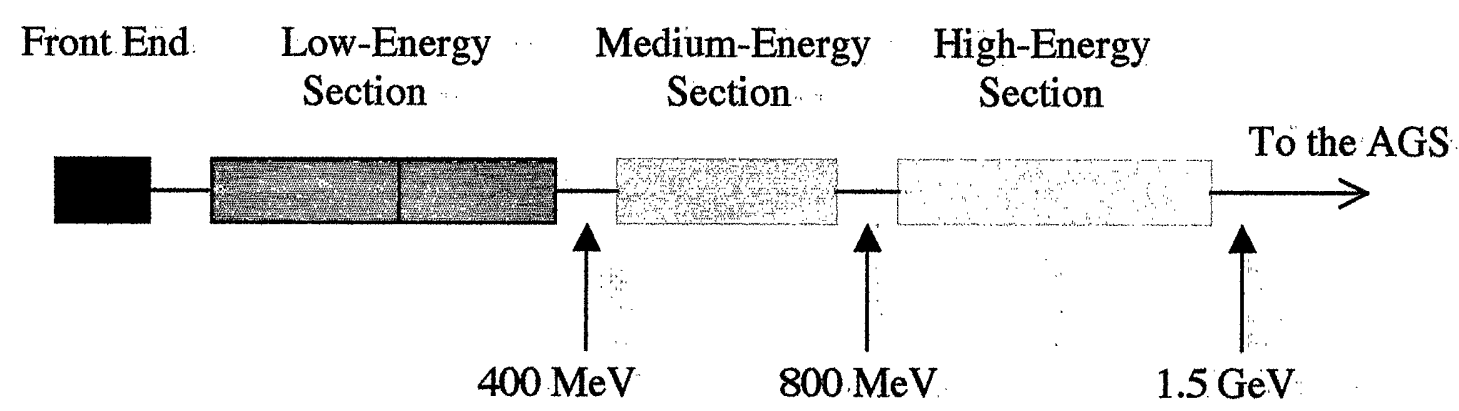

Figure 1. Layout of the 1.5-GeV Super-Conducting Linac for the AGS

\section{AGS Performance with the New Injector}

The AGS performance with the new injector is summarized in Table 1. The average beam current of $45 \mu \mathrm{A}$ yields the average beam power of $1 \mathrm{MW}$ at the top energy of 24 $\mathrm{GeV}$. The repetition rate of 2.5 beam pulses per second is assumed, that gives an intensity of $1 \times 10^{14}$ protons accelerated per AGS cycle. At the end of injection, that takes about. 300 turns, with a Linac pulse length of about $1 \mathrm{~ms}$, the space-charge tune depression is $\Delta v=0.2$, assuming a bunching factor (the ratio of beam peak current to average current), during the early part of the acceleration cycle, of 4 . Also, with a normalized beam emittance of $250 \pi \mathrm{mm}$-mrad; the actual beam emittance is $100 \pi \mathrm{mm}$-mrad at $1.5 \mathrm{GeV}$. Obviously; the effective acceptance of the AGS at injection is to be larger than this beam emittance value. Conversely, a higher tune-depression may result for a smaller beam emittance. The beam duty cycle is estimated at $0.25 \%$, but the Linac may need to operate at least twice that value, that is a duty cycle of $0.5 \%$.

\section{The Front End}

The layout of the Front End of the Linac is shown in Figure 2. It is made of an ion source operating with about $1 \%$ duty cycle at the repetition rate of 2.5 pulses per second. The beam current within a pulse is $37.5 \mathrm{~mA}$ of negative-hydrogen ions. The ion source seats on a platform at $35-50 \mathrm{kVolt}$, and is followed by a $750-\mathrm{keV}$ RFQ that works at 201.25 MHz. The beam is pre-chopped by a chopper located between the ion source and the RFQ. The beam chopping extends over $60 \%$ of the beam length, at a frequency matching the accelerating $\mathrm{RF}(2.06 \mathrm{MHz}$, assuming an harmonic number $\mathrm{h}=6)$ at injection into the AGS. Moreover, the transmission efficiency through the RFQ is taken conservatively to be $80 \%$, so that the average current within the beam pulse in the Linac, where we assume no further beam loss, is $18 \mathrm{~mA}$, with a peak value of $30 \mathrm{~mA}$.

The combination of the chopper and of the RFQ pre-bunches the beam with a sufficiently small longitudinal extension so that each of the beam bunches at $201.25 \mathrm{MHz}$ can be entirely fitted in the accelerating RF buckets of the following Low-Energy section. At this purpose a buncher, operating at the same frequency $201.25 \mathrm{MHz}$, is located between the Front End and the Low-Energy section. 


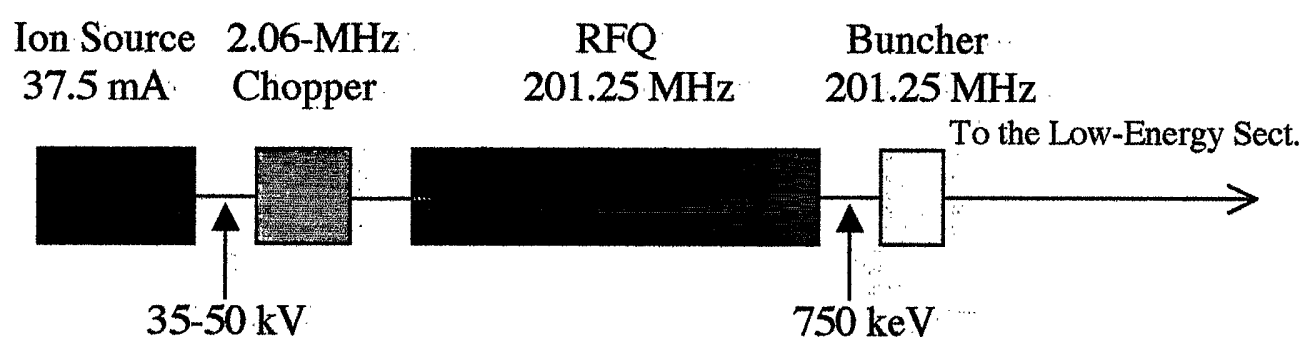

Figure 2. Schematics of the Front-End

Because of the higher repetition rate, the present pre-injector (Ion Source and RFQ) cannot be used, and all the components of the Front End are to be built anew. But none of them represent serious technical challenge since the required performance is well within the demonstrated technology. The cost for the Front End is expected in a couple of million dollars.

\section{The Low-Energy Section}

The Low-Energy section of the SCL is shown schematically in Figure 3. It is made of two parts. The first part includes the initial five tanks of the present 200-MeV AGSLinac. This is a room-temperature device operating at $201.25 \mathrm{MHz}$ that presently can operate with a slow duty cycle of less than $0.1 \%$. It is important to demonstrate that it can be made to operate at the proposed extended duty cycle of half percent. The first five tanks of the AGS-Linac accelerate to $116 \mathrm{MeV}$. The second part is an upgrade similar.to that done for the original Fermilab Linac. The last four tanks are to be replaced with cavities operating at $805 \mathrm{MHz}$ and thus with a more compact accelerating gradient. The final energy would then be $400 \mathrm{MeV}$, instead of the original $200 \mathrm{MeV}$, achieved essentially over the same length. In our case there are two options: a room-temperature Drift-Tube Linac (DTL) similar to the one adopted at Fermilab, and a super-conducting. option. Both would operate at $805 \mathrm{MHz}$, have an average gradient of $3.8 \mathrm{MeV} / \mathrm{m}$, have about the same length of $75 \mathrm{~m}$, and share about the same cost of about $40 \mathrm{M} \$$. The final choice may depend on the results of a more detailed study. Here we adopt the superconducting solution that will be described below.

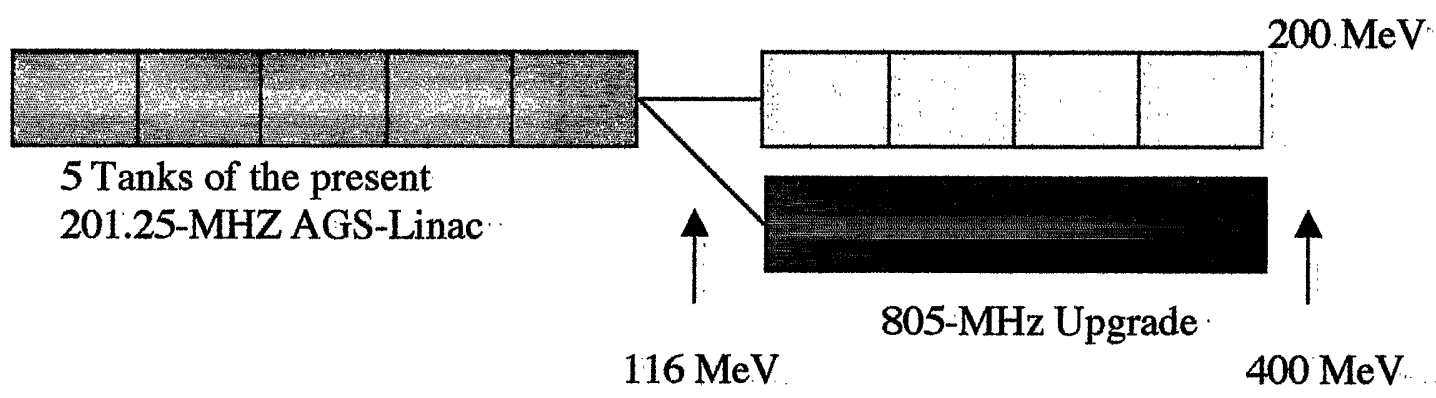

Figure 3. The SCL Low-Energy Section 
Table 1. Injector and AGS Parameters for the Upgrade

\begin{tabular}{|l|r|}
\hline Linac Average Power, $\mathrm{kW}$ & 62.5 \\
\hline \hline Kinetic Energy, GeV & 1.5 \\
\hline$\beta$ & 0.9230 \\
\hline Momentum, GeV/c & 2.2505 \\
\hline Magnetic Rigidity, T-m & 7.5068 \\
\hline Repetition Rate, $\mathrm{Hz}$ & 2.5 \\
\hline Average Current, $\mu \mathrm{A}$ & 45 \\
\hline Number of Protons $/$ pulse & $1.0 \times 10^{14}$ \\
\hline AGS Circumference, $\mathrm{m}$ & 807.12 \\
\hline Revol. Frequency, MHz & 0.3428 \\
\hline Revolution Period; $\mu \mathrm{s}$ & 2.9169 \\
\hline Bending Radius & 79.832 \\
\hline Injection Field & 0.9403 \\
\hline Ion Source Current, $\mathrm{mA}$ & 37.5 \\
\hline RFQ Transmission; $\%$ & 80 \\
\hline Chopping Ratio, \% & 60 \\
\hline Peak Current, mA & 30 \\
\hline Average Current, $\mathrm{mA}$ & 18 \\
\hline Protons per Turn & $3.3 \times 10^{11}$ \\
\hline Number of injected Turns & $\sim 300$ \\
\hline Beam Pulse Length, ms & 1.0 \\
\hline Linac Duty Cycle, \% & 0.5 \\
\hline Bunching Factor & 4 \\
\hline Norm. Emitt., $\pi$ mm-mrad & 250 \\
\hline Emittance, $\pi$ mm-mrad & 100 \\
\hline Space-Charge $\Delta v$ & 0.20 \\
\hline & \\
\hline
\end{tabular}

\section{The Medium- and High-Energy Sections}

Both of these sections are super-conducting and operate at $1,620 \mathrm{MHz}$. This frequency is four times that of the Low-Energy section. A higher accelerating RF is desirable for obtaining a larger accelerating gradient, with a more compact structure and shorter length, and for reduced cost. There is a limit nonetheless due to the dilution of the accelerating gradient caused by the transit time factors, and by the reduction of the internal aperture where the beam is accommodated and accelerated.

The Medium-Energy section accelerates from 400 to $800 \mathrm{MeV}$. The High-Energy section takes the beam to $1.5 \mathrm{GeV}$. The division of the SCL in more than one section is required to minimize the number of cavities and cells with different length. In fact, in the case of acceleration of protons, the velocity of the particles $\beta$ varies considerably from one end to the other. For an optimum accelerating gradient, the length $L$ of the cavity cell should be adjusted so that 


$$
L=\beta \lambda / 2
$$

where $\lambda$ is the RF wavelength. Clearly; if one takes $\lambda$ constant, the cell length $L$ has to be adjusted continuously from one cavity to the next, as also $\beta$ varies. This may require too many cavities of different size that could make the construction of the Linac cumbersome and expensive. We propose here [3] that each of the Linac sections are made of the same number of cavities with the same number of cells of fixed length. The number of cavities and cells, and length is different from one section to the next. In each section the cell length is designed around a different central value $\beta_{0}$. These design considerations apply to the Medium- and High-Energy sections, but also to the super-conducting part of the Low-Energy section.

\section{Design of the Super-Conducting Linac}

The configuration and the design procedure of the SCL are described in detail in [3]. It is typically a sequence of a number of identical periods as shown in Figure 4. Each period is made of a cryo-module of length $L_{\text {cryo }}$ and of an insertion of length $L_{\text {ins }}$. The insertion is needed for the placement of focusing quadrupoles, vacuum pumps, steering magnets, beam diagnostic devices, bellows and flanges. It can be either at room temperature or in a cryostat as well. Here we assume that the insertions are at room temperature. The cryomodule includes $M$ identical cavities; each of $N$ identical cells; and each having a length $N L_{\text {cell }}$, where $L_{\text {cell }}$ is the length of a cell. Cavities are separated from each other by a drift space $d$. An extra drift of length $L_{\mathrm{w}}$ may be added internally on both sides of the cryomodule to provide a transition between cold and warm regions. Thus;

$$
L_{\text {cyyo }}=M N L_{\text {cell }}+(M-1) d+2 L_{\mathrm{w}}
$$

There are two symmetric intervals: a minor one, between the two middle points A and $\mathrm{B}$, as shown in Figure 4, that is the interval of a cavity of length $N L_{\text {cell }}+d$; and a major one, between the two middle points $C$ and $D$, that defines the range of a period of total length $L_{\text {cryo }}+L_{\text {ins }}$. Thus, the topology of a period can be represented as a drift of length $g$, followed by $M$ cavity intervals, and a final drift of length $g$, where

$$
g=L_{\mathrm{w}}+\left(L_{\mathrm{ins}}-d\right) / 2
$$

The choice of cryo-modules with identical geometry, and with the same cavity/cell configuration, is economical and convenient for construction. But there is, nonetheless; a penalty due to the reduced transit-time-factors when a particle crosses cavity cells, with length adjusted to a common central value $\beta_{0}$ that does not correspond to the particle instantaneous velocity, as given by Eq. (1).

The major parameters of the three sections of the SCL are given in Tables 2 and 3. The cost estimate (with no contingency) for each section of the SCL has been made assuming the cost and if parameters shown in Table 4 . The total expected cost is around $100 \mathrm{M} \$$ : The total length of the Medium- and High-Energy sections combined is about. $120 \mathrm{~m}$. This is to be fit between the end of the present AGS-Linac and the injection point 
to the AGS. In case a straight pattern is not possible, the two sections can be turned at an angle with respect to each other.
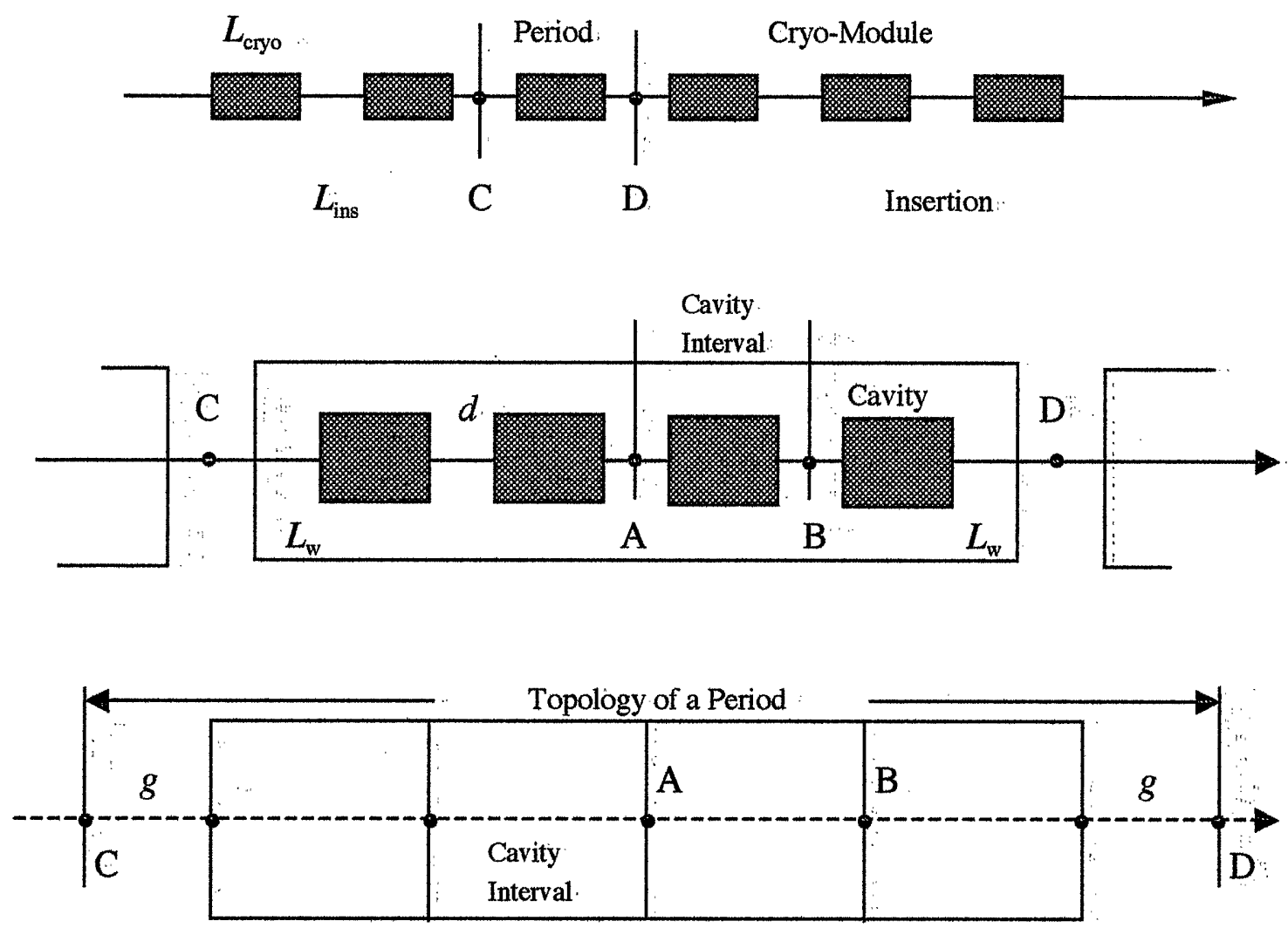

Figure 4. Configuration of a Proton Super-Conducting Linear Accelerator

\section{Other Design Considerations}

The length of the Linac depends on the average accelerating gradient. The local gradient has a maximum value that is limited by three causes: (1) The surface field limit at the frequency of $805 \mathrm{MHz}$ is $26 \mathrm{MV} / \mathrm{m}$. For a realistic cavity shape, we set a limit of a 13 $\mathrm{MV} / \mathrm{m}$ on the axial electric field. In the following two sections; the surface field limit at $1,620 \mathrm{MHz}$ is $40 \mathrm{MV} / \mathrm{m}$, correspondingly we adopt a limit of a $20 \mathrm{MV} / \mathrm{m}$ on the axial electric field. (2) There is a limit on the power provided by rf couplers that we take here not to exceed $400 \mathrm{~kW}$, including a contingency of $50 \%$ to avoid saturation effects. (3) To make the longitudinal motion stable, we can only apply an energy gain per cryo-module that is a relatively small fraction of the beam energy in exit of the cryo-module. The conditions for stability of motion have been derived in [3]:

The proposed mode of operation is to operate each section of the SCL with the same RF input power per cryo-module. This will result to some variation of the actual axial field from one cryo-module to the next: If one requires also a constant value of the axial field; this may be obtained by adjusting locally the value of the RF phase angle that has been taken to be nominally $30^{\circ}$. 
It is possible to build the entire SCL in two stages. During the first stage, which includes the Medium- but not the High-Energy section, the final energy if $0.8 \mathrm{GeV}$. In a second stage the High-Energy section is added for the final energy of $1.5 \mathrm{GeV}$, if indeed this should result to be necessary. Conversely, the fifteen cryo-modules can be added sequentially, one after the other, as many as required, provided that the external focusing elements have already been provided to transport the beam for injection into the AGS.

Table 2. General Parameters of the SCL

\begin{tabular}{|c|c|c|c|}
\hline Linac Section & $\mathrm{LE}$ & ME & $\mathrm{HE}$ \\
\hline Average Beam Power, $\mathrm{kW}$ : & 18 & 36 & 62.5 \\
\hline Average Beam Current, $\mu \mathrm{A}$ & 45 & 45 & 45 \\
\hline Initial Kinetic Energy, $\mu \mathrm{eV}$ & 116 & 400 & 800 \\
\hline Final Kinetic Energy, $\mu \mathrm{eV}$ & 400 . & 800 & 1500 \\
\hline Frequency, $\mathrm{MHz}$ & 805 & 1620 & 1620 \\
\hline No. of Protons / Bunch $\times 10^{8}$ & 9.32 & 9.32 & 9.32 \\
\hline Temperature, ${ }^{\circ} \mathrm{K}$ & 2.0 & $2: 0$ & 2.0 \\
\hline Cells / Cavity & 4 & 8 & 8 \\
\hline Cavities / Cryo-Module & 4 & 4 & 4 \\
\hline Cavity Separation, cm & 32 & 16 & 16 \\
\hline Cóld-Warm Transition, $\mathrm{cm}$ & 30 & 30 & 30 \\
\hline Cavity Internal Diameter, cm: & 10 & 5 & 5 \\
\hline Length of Warm Insertion, $m$ & 1.079 & 1.079 & 1.079 \\
\hline Accelerating Gradient, $\mathrm{MeV} / \mathrm{m}$ & 11.9 & 22.0 & 21.5 \\
\hline Cavities / Klystron & 4 & 4 & 4. \\
\hline No. of rf Couplers / Cavity $\ldots$ & 1. & 1 & 1 \\
\hline Rf Phase Angle & $30^{\circ}$ & $30^{\circ}$ & $30^{\circ}$ \\
\hline Method for Transverse Focussing & FODO & FODO & FODO \\
\hline Betatron Phase Advance / FODO cell & $90^{\circ}$ & $90^{\circ}$ & $90^{\circ}$ \\
\hline Norm. rms Emittance, $\pi \mathrm{mm}$-mrad & 0.30 & 0.30 & 0.30 \\
\hline Rms Bunch Area, $\pi^{\circ} \mathrm{MeV}$ & 0.5 & 0.5 & 0.5 \\
\hline
\end{tabular}

Negative ion stripping during transport down the SCL has been found to be very negligible. But the final bend, before injection into the AGS, could be of a concern [4]. To control the rate of beam loss by stripping to a $10^{-4}$ level, the bending field should not exceed $2.6 \mathrm{kGauss}$ over a total integrated bending length of $0.5 \mathrm{~m}$ for each degree of bend.

A program [5] was written in Visual Basic included with the MS Excel application, to calculate the beam and rf dynamics during acceleration in each of the four sections of the SCL. The results are displayed in Figures 6 to 14 .

Transverse focusing of the beam is provided with alternating gradient quadrupoles located in the warm insertions. The arrangement chosen is the one of a sequence of FODO cells. 
Table 3. Summary of the SCLDesign

\begin{tabular}{|c|c|c|c|}
\hline Linac Section & LE & $\mathrm{ME}$ & $\mathrm{HE}$ \\
\hline Velocity, $\beta$ : in & 0.4560 & 0.7131 & 0.8418 \\
\hline out: & 0.7131 & 0.8418 & 0.9230 \\
\hline Cell Reference $\beta_{0}$ & 0.520 & 0.750 & 0.865 \\
\hline Cell Length, $\mathrm{cm}$ & 9.68 & 6.98 & 8.05 \\
\hline Total No of Periods. & 18 & 10 & 15 \\
\hline Length of a period, $m$ & 4.118 & 4.393 & 4.736 \\
\hline FODO-Cell ampl. func., $\beta_{\mathrm{o}}, \mathrm{m}$ & 14.30 & 15.00 & 16.17 \\
\hline Total Length, $m$ & 75.39 & 43.93 & $71: 04$ \\
\hline Coupler rf Power, $\mathrm{kW}\left({ }^{*}\right)$ & 120 & 320 & 360 \\
\hline Energy Gain/Period, $\mathrm{MeV}$ & 16.00 & 42.67 & 48.00 \\
\hline Total No. of Klystrons & 18 & 10 & 15 \\
\hline Klystron Power, $\mathrm{kW}\left({ }^{*}\right)$ & 720 & 1920 & 2160 \\
\hline $\mathrm{Z}_{0} \mathrm{~T}_{0}^{2}, \mathrm{ohm} / \mathrm{m}$ & 261.6 & $\overline{544.2}$ & 723.9 \\
\hline$Q_{0} \times 10^{9}$ & 5.4 & 5.2 & 5.9 \\
\hline Ave. Dissipated Power, $\mathrm{kW}$ & 0.014 & 0.014 & 0.012 \\
\hline Ave. HOM-Power, $\mathrm{kW}$ & 0.0005 & 0.0012 & 0.0018 \\
\hline Ave. Cryogenic Power, $\mathrm{kW}$ & 0.294 & 0.181 & 0.288 \\
\hline Ave. Beam Power, MW & 0.013 & 0.018 & 0.032 \\
\hline Total Ave. rf Power, MW ( $*$ ) & 0.031 & 0.034 & 0.055 \\
\hline Ave. AC Power for if, MW (*) & 0.068 & 0.075 & 0.122 \\
\hline Ave. AC Power for Cryo., MW & 0.074 & 0.045 & 0.072 \\
\hline Total Ave. AC Power, MW (*) & 0.142 & 0.121 & 0.194 \\
\hline Efficiency, $\%(*)$ & 9.0 & 14.9 & 16.2 \\
\hline $\begin{array}{r}\text { Capital Cost'00 M\$: } \\
\text { Rf Klystron (*) }\end{array}$ & 0.076 & 0.085 & 0.137 \\
\hline Electr. Distr. (*) & 0.020 & 0.017 & 0.027 \\
\hline Refrig. Plant & 0.589 & 0.362 & 0.577 \\
\hline Warm Structure & 3.075 & $1.780^{\circ}$ & 2.590 \\
\hline Cold Structure & 27.983 & 16.572 & $27: 428$ \\
\hline Tunnel- & 7.647 & 4.501 & 7.212 \\
\hline Total Cost, '00 M\$ (*) & 39.390 & 23.318 & 37.971 \\
\hline Operation Cost,, $00 \mathrm{M} \$ / \mathrm{y}\left(^{*}\right)$ & 0.046 & 0.040 & 0.064 \\
\hline
\end{tabular}

${ }^{*}$ Including $50 \%$ rf power contingency.

\section{RF Power Distribution}

One of the advantages of the SCL is that cavities can be operated independently from each other provided that the RF power in input can be controlled both in phase and amplitude. Even in the case one cavity (or a cell within) should fail, it is in principle 
possible to bridge it across and still let the beam through to next cavity. At this purpose, though, it is important that the RF power from a source like a klystron is not split too many times, as shown in Figure 5. We have adopted four cavities under one klystron and a single coupler feeding an individual cavity:

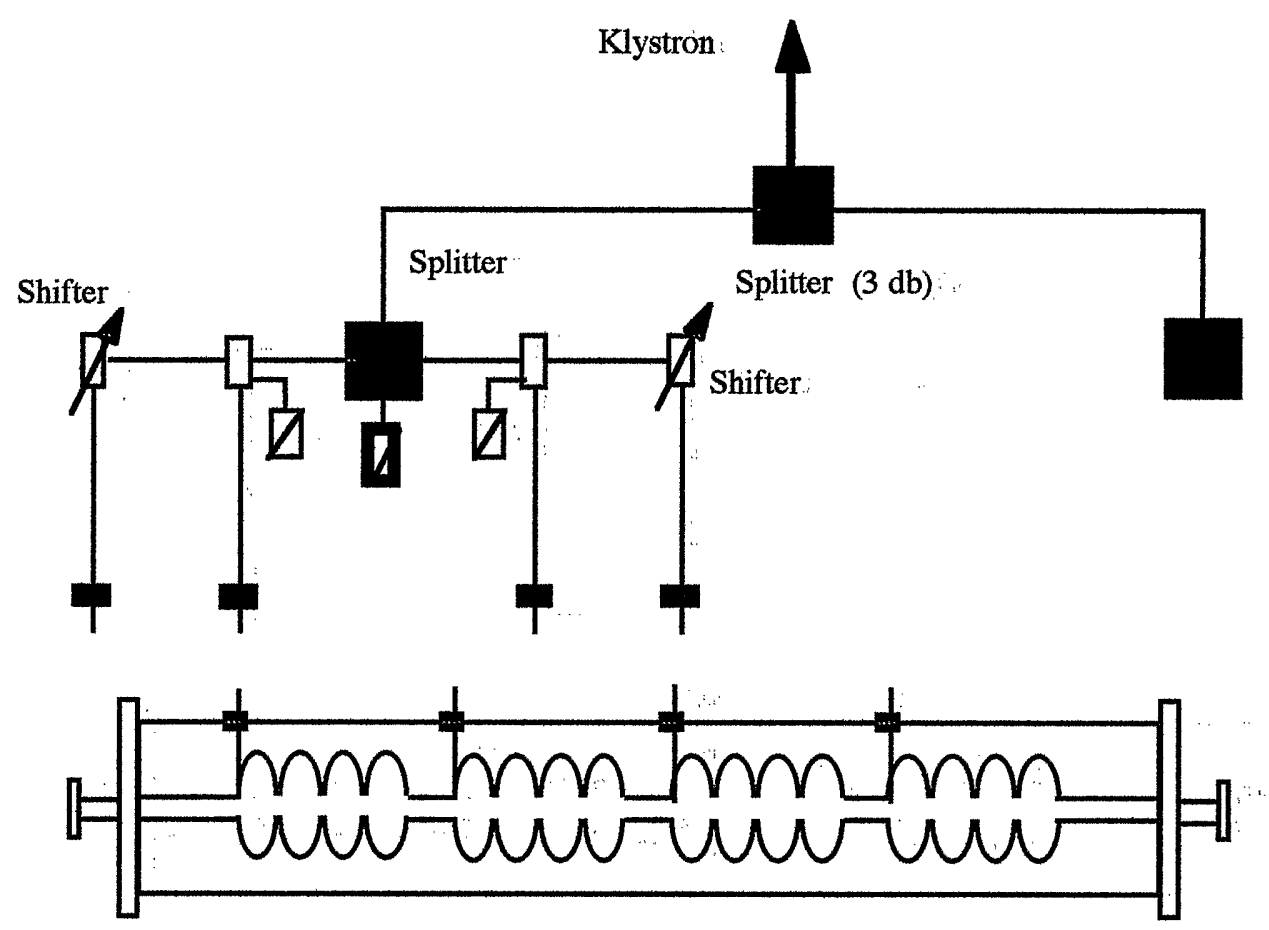

Figure 5: RF Power Distribution

\section{Pulsing a Super-Conducting Linac}

A SCL is most advantageous for a continuous mode of operation. There are two problems in the case of the pulsed-mode of operation. First, the pulsed thermal cycle introduces Lorentz forces that deform the cavity cells out of resonance. This can be controlled with a thick cavity wall strengthened to the outside by supports. Second, there is an appreciable period of time to fill the cavities with RF power before the maximum gradient is reached [3]. During the filling time, extra power is dissipated also before the beam is injected into the Linac. The extra amount of power required is the ratio of the filling time to the beam pulse length. The filling time is $0.59 \mathrm{~ms}$ for the LE section, $0.25 \mathrm{~ms}$ for the ME section,. and $0.16 \mathrm{~ms}$ for the HE section. In comparison, the beam pulse length is $1.0 \mathrm{~ms}$.

\section{Conclusion}

We have done a preliminary study of a Super-Conducting Linac as a new injector to the AGS. The study has two main features that are to be taken into account to assess closely 
the performance and the usefulness of the accelerator. First; the beam power is very modest; that causes the efficiency of the Linac to be rather low, as shown in Table 3. Usually, high efficiency in a SCL is obtained in the case of acceleration of beams with large intensity. Secondly, as also shown in Table 3 , the cost is essentially given by the manufacturing of the cry-modules, that is, cavities and cryostats with their interface. The cost of the RF in comparison is very modest. Because of the relatively low beam and RF. power, the expected cost of the yearly operation is also rather low.

Table 4. Cost.('00 \$) and Other Parameters

\begin{tabular}{|l|r|l|}
\hline AC-to-rf Efficiency & 0.45 & For pulsed mode \\
\hline Cryogenic Efficiency & 0.004 & At $2.0^{\circ} \mathrm{K}$ \\
\hline Electricity Cost & 0.05 & $\$ / \mathrm{kWh}$ \\
\hline Linac Availability & 75 & $\%$ of yearly time \\
\hline Normal Conducting Cost & 150 & $\mathrm{k} \$ / \mathrm{m}$ \\
\hline Superconducting Cost & 500 & $\mathrm{k} \$ / \mathrm{m}$ \\
\hline Tunnel Cost & 100 & $\mathrm{k} \$ / \mathrm{m}$ \\
\hline Cost of Klystron & 2.50 & $\$ / \mathrm{W}$ of rf Power \\
\hline Cost of Refrigeration Plant & 2 & $\mathrm{k} \$ / \mathrm{W}$ of Power @ $2.0^{\circ} \mathrm{K}$ \\
\hline Cost of Electrical Distribution & 0.14 & $\$ / \mathrm{W}$ of AC Power \\
\hline
\end{tabular}

\section{References}

[1]:D. Raparia and A.G. Ruggiero, "A Super-Conducting Linac Injector to the BNL-AGS”. BNL-Internal Report C-A/AP39. July 2000.

[2] D. Raparia and A.G. Ruggiero, "A Super-Conducting Linac Injector to the BNL-AGS". Presented to the XX International Linac Conference, Montrey; California. August 21-25, 2000.

[3] A. G. Ruggiero, "Design Considerations on a Proton Superconducting Linac". BNL-Internal Report 62312. August 1995.

[4]:A. G. Ruggiero, "Negative-Ion Injection by Charge Exchange at $2: 4 \mathrm{GeV}$ ". BNL-Internal Report 62310. September 1995.

[5] The program is available by making request to one of the Authors. 

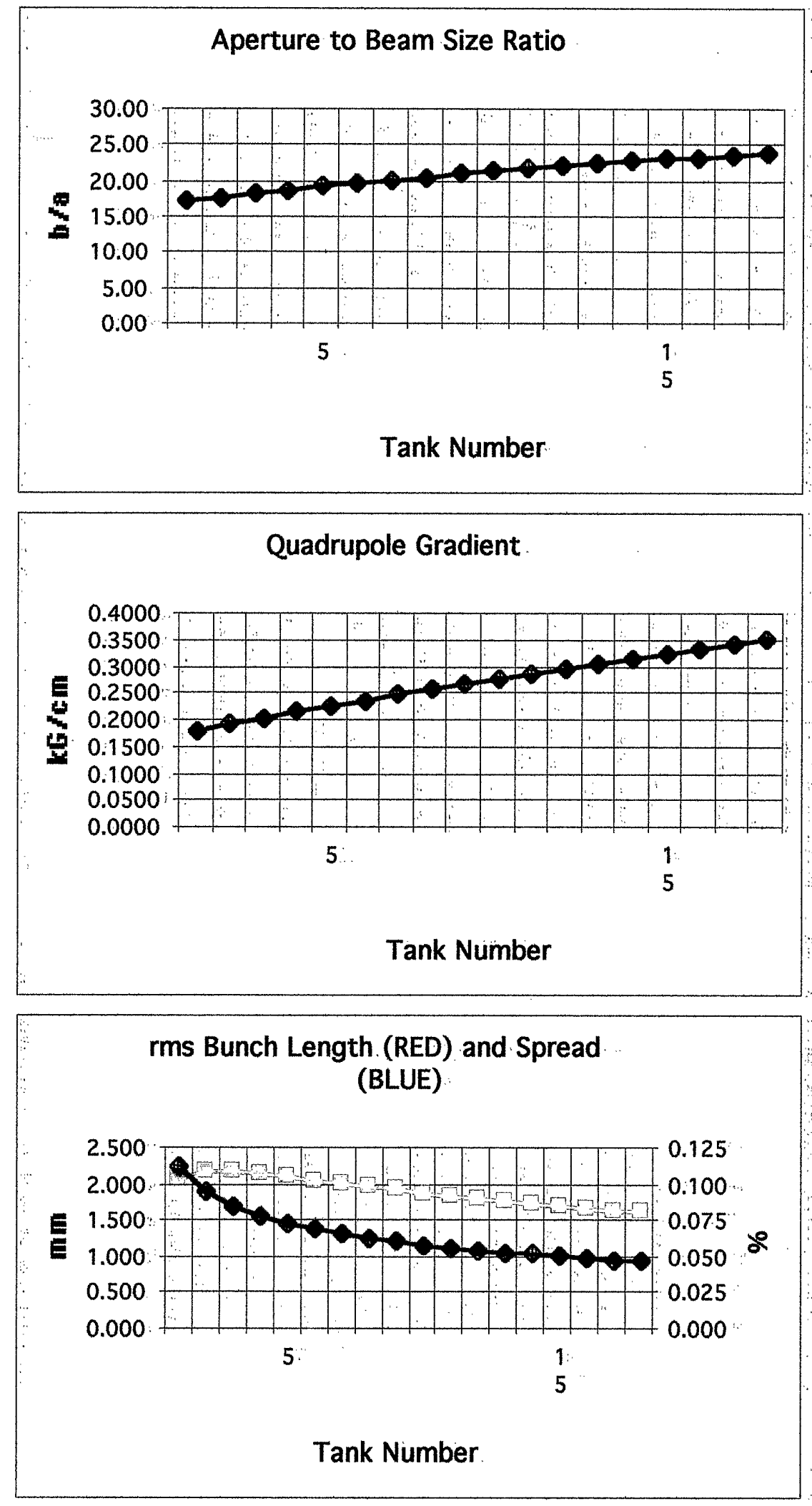

Figure 6. Plots (1) of Behavior vs. period (tank) number of LESection. 

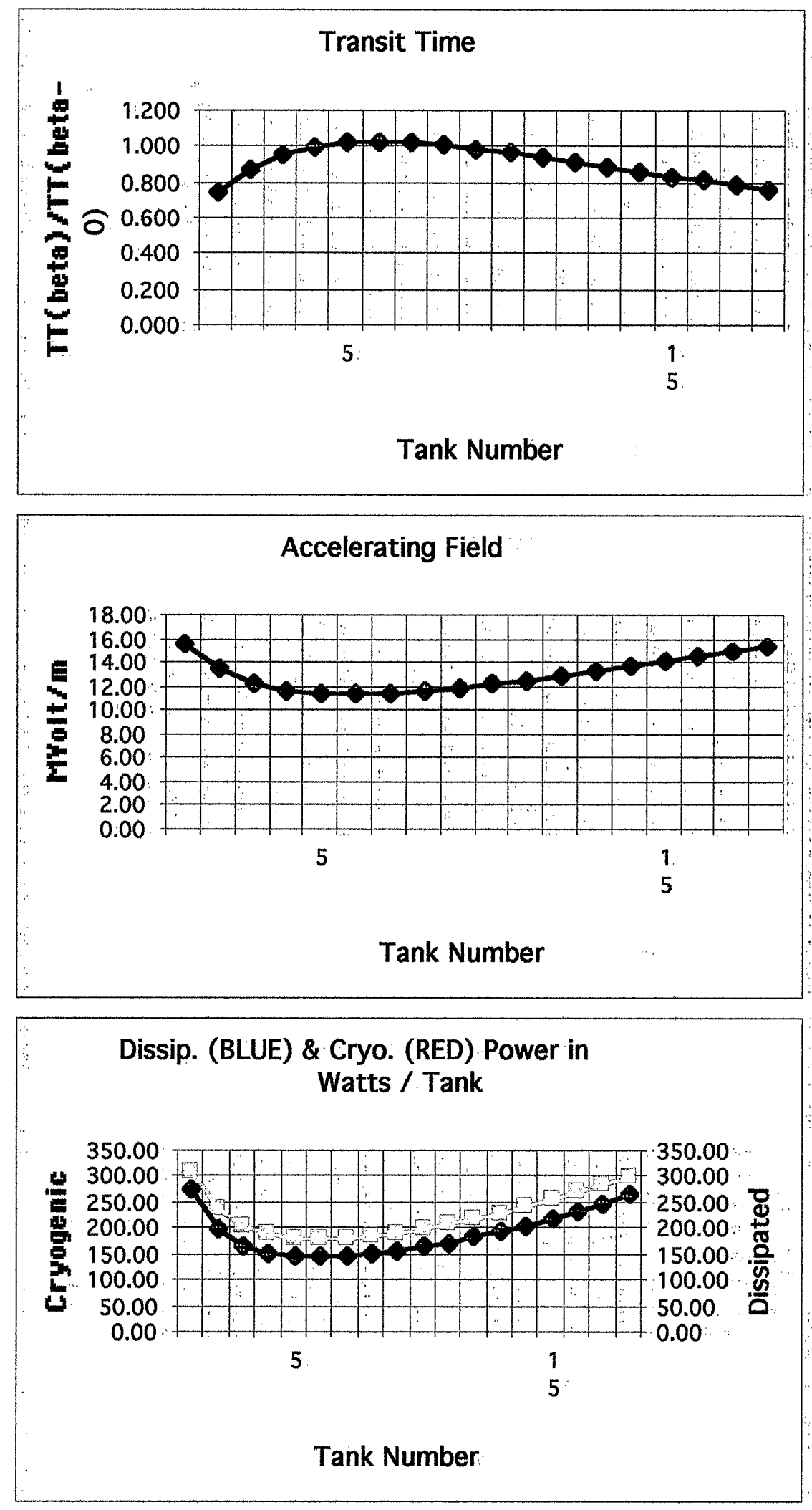

Figure 7. Plots (2) of Behavior vs. period (tank) number of LE Section. 

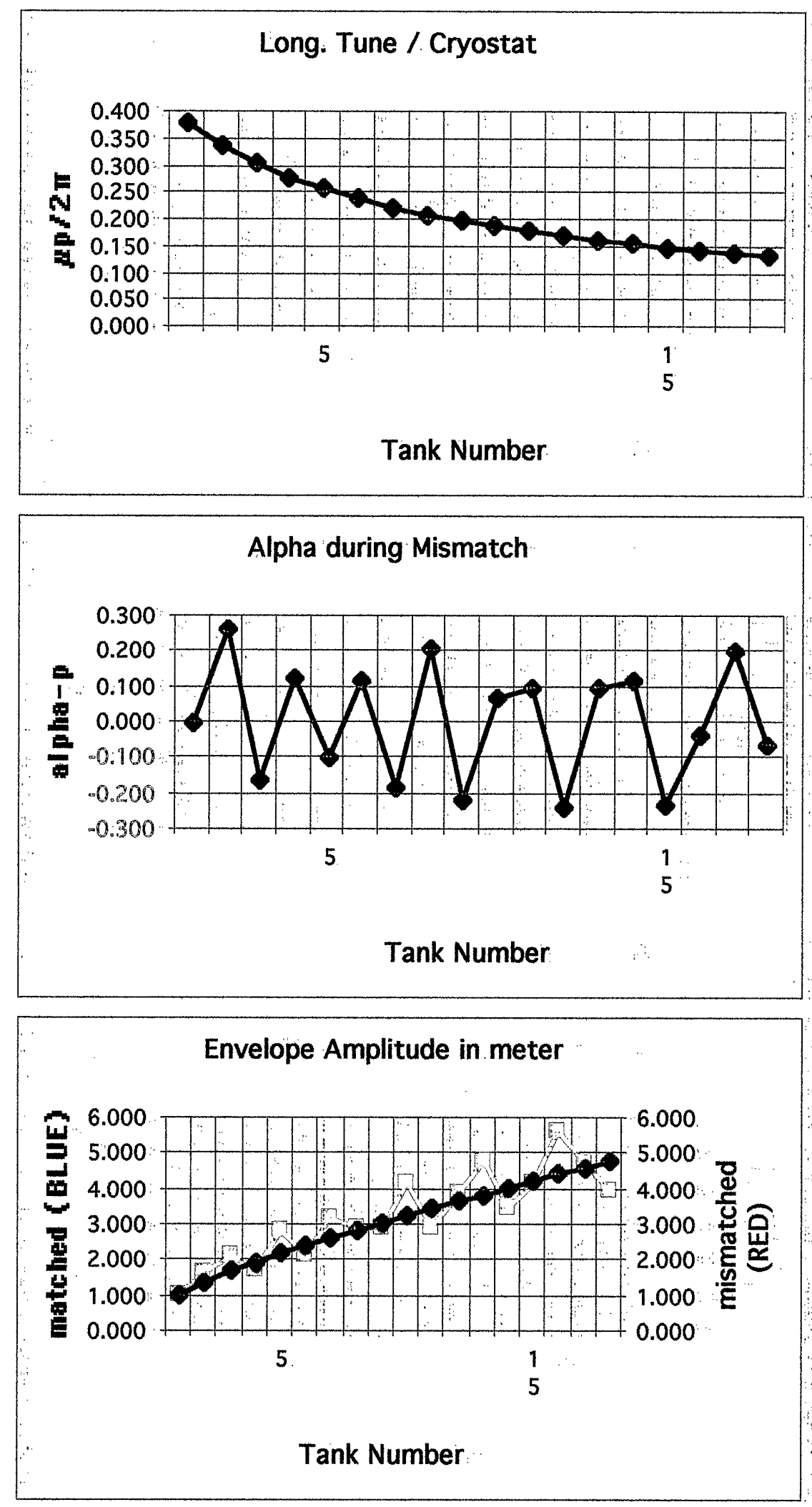

Figure 8. Plots (3) of Behavior vs. period (tank) number of LE Section. 

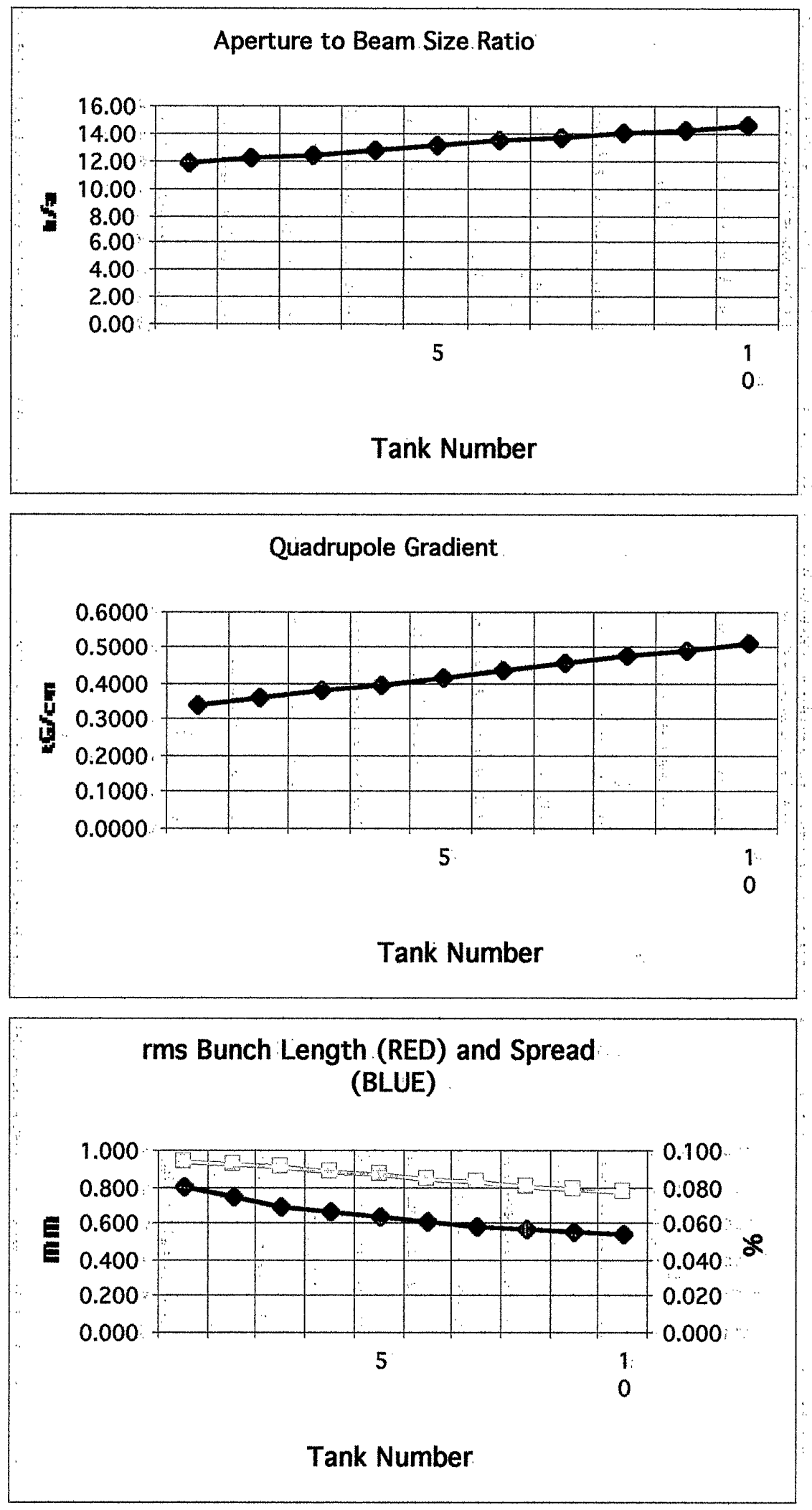

Figure 9. Plots (1) of Behavior vs. period (tank) number of ME Section. 

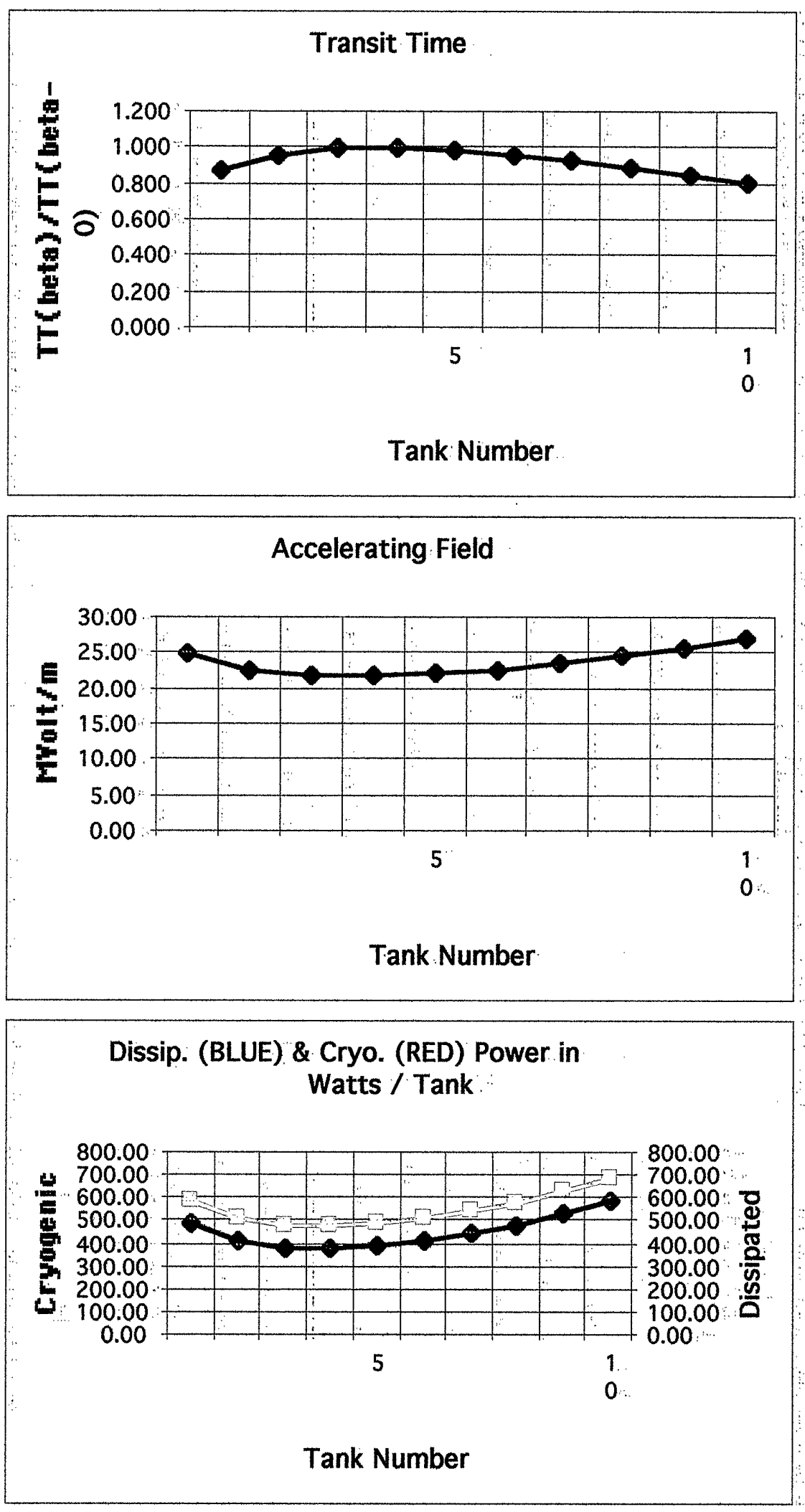

Figure 10: Plots (2) of Behavior vs. period (tank) number of ME Section. 

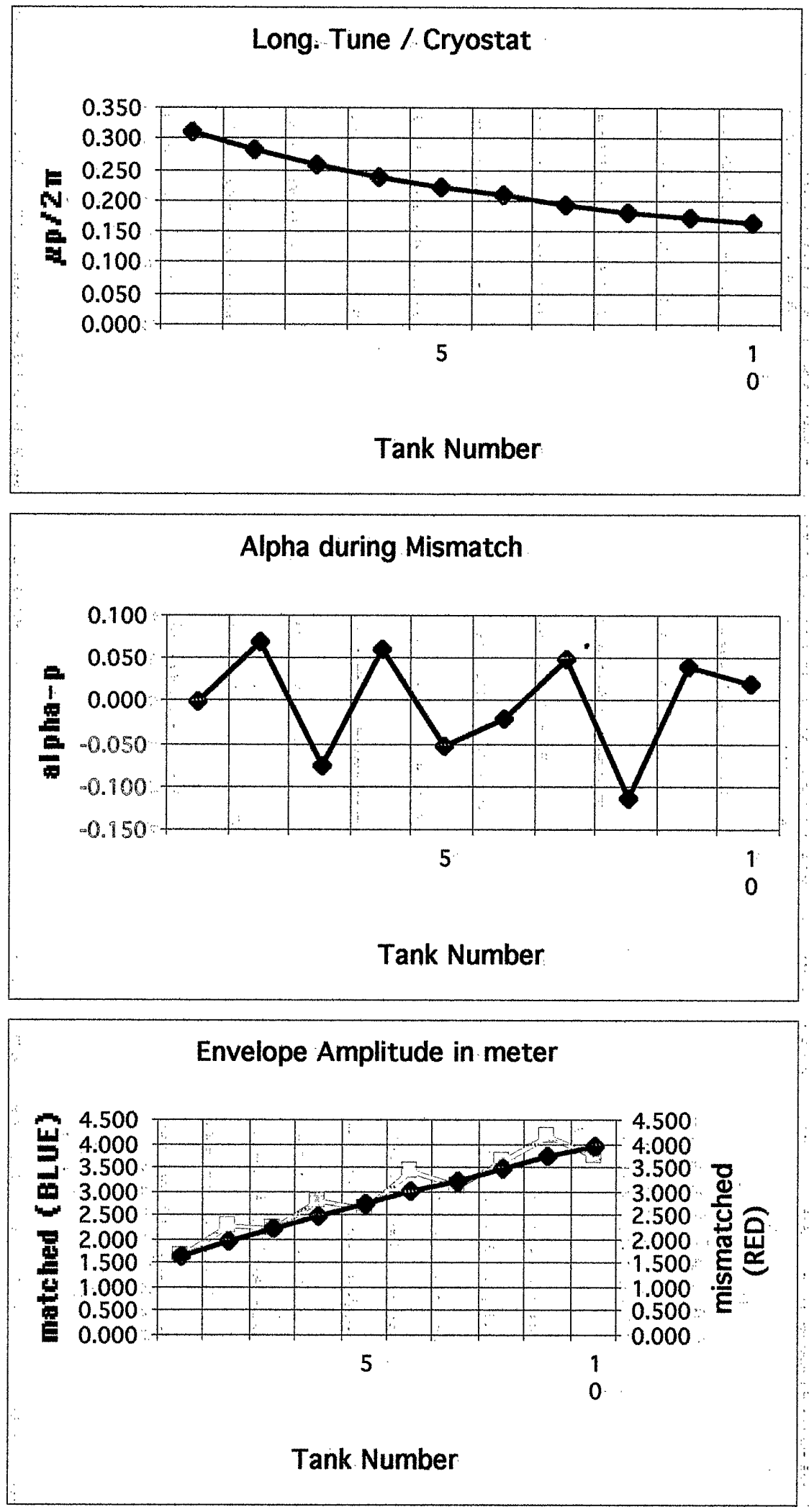

Figure 11: Plots (3) of Behavior vs. period (tank) number of ME Section. 

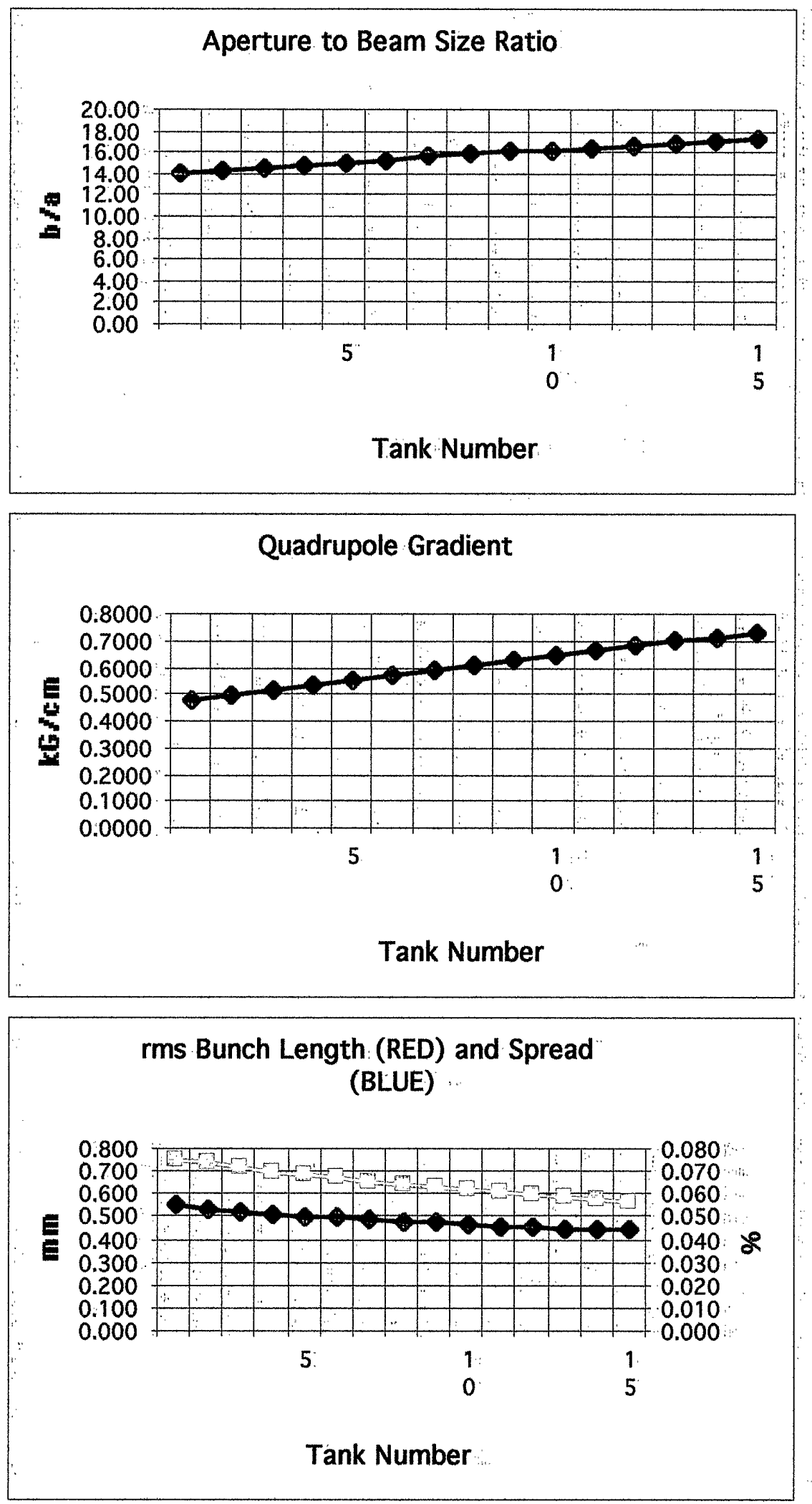

Figure 12. Plots (1) of Behavior vs. period (tank) number of HE Section. 

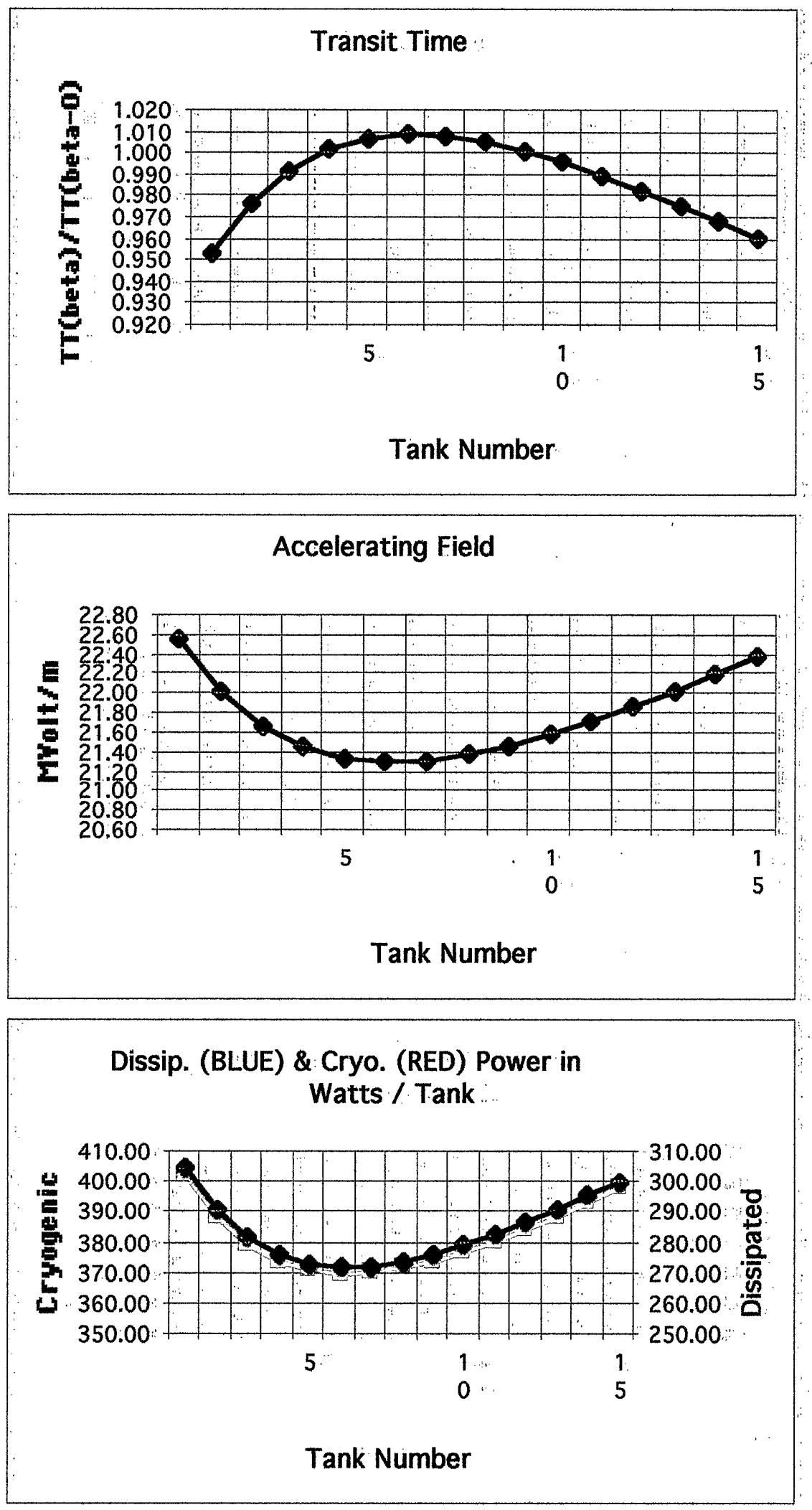

Figure 13. Plots (2) of Behavior vs. period.(tank) number of HE Section. 

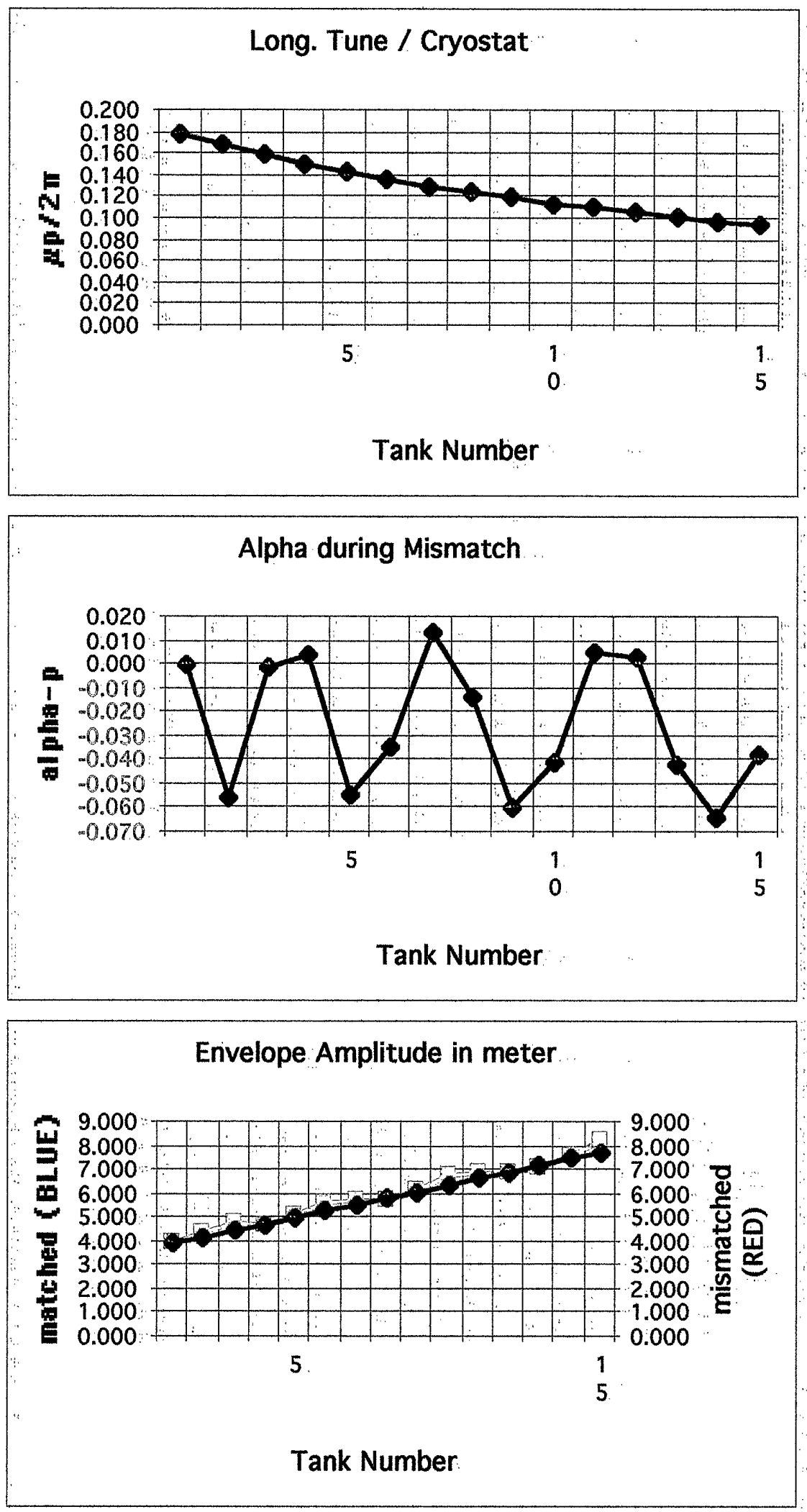

Figure 14. Plots (3) of Behavior vs. period (tank) number of HE Section. 